\title{
Gestión de documentación multimedia con Synchronized Multimedia Integration Language (SMIL)
}

\author{
Blanca Gil Urdiciain \\ José Ramón Pérez Agüera \\ Rodrigo Sánchez Jiménez \\ Universidad Complutense de Madrid (España)
}

\subsection{Resumen}

Se analiza el módulo de metadatos del Synchronized Multimedia Integration Language (SMIL) de cara a su utilización para el tratamiento de documentación multimedia. A continuación se describe un ejemplo práctico en un entorno real. Finalmente, se establecen unas conclusiones que demuestran la utilidad de SMIL a nivel profesional y, fundamentalmente, en industrias relacionadas con la documentación audiovisual.

Palabras clave: Synchronized Multimedia Integration Language (SMIL). Extensible Markup Language (XML). Metadatos. Recuperación de información. Documentación multimedia. Documentación audiovisual.

\subsection{Abstract}

The metadata module of Synchronized Multimedia Integration Language (SMIL) is analyzed in order to apply it to the indexing and abstracting of multimedia documentation. After this, an application of this language in a real environment is presented as example. Finally, the utility of SMIL in a professional level, mainly in industries related to audiovisual documentation, is established.

Keywords: Synchronized Multimedia Integration Language (SMIL). Extensible Markup Language (XML). Metadata. Information retrieval. Multimedia documentation. Audiovisual documentation.

\section{Introducción}

El camino hacia la Web Semántica pasa por la identificación unívoca, clara y concisa de cada una de las unidades informativas que la componen. En el caso de la documentación multimedia esto es especialmente delicado, ya que cada documento puede estar compuesto a su vez por otros documentos que - si seguimos 
la afirmación anterior - deben a su vez poder ser identificados tanto por separado como en conjunto de cara a su recuperación posterior. La identificación de estos documentos se complica en función del formato en el que se encuentran codificados. Así, por ejemplo, un documento HTML puede llevar consigo sus propios metadatos, que ayudan a su recuperación mediante buscadores y aglutinan la metainformación suficiente que se refiere a ese elemento en concreto. En el caso de documentos de video o audio esta capacidad de autodescripción se complica, haciendo necesaria la realización de esfuerzos a este respecto, de los cuales han surgido varias iniciativas entre las cuales destaca MPEG-7. Ahora bien, ¿que ocurre en el caso de un documento multimedia, donde texto, video y audio se reúnen para construir un discurso determinado que depende fundamentalmente de la combinación de documentos en distintos formatos? El objetivo de este trabajo consiste en intentar dar respuesta a esta pregunta y mostrar una solución al problema de la descripción de elementos multimedia en formato electrónico.

El auge de lo multimedia ha acompañado a Internet desde su creación. De hecho, gran parte de las mejoras técnicas que han acontecido en la Red en los últimos años han ido encaminadas en esta dirección. Productos comerciales como Flash y Director de Macromedia han proporcionado las herramientas necesarias para la composición de mensajes multimedia, los cuales incluso han traspasado los límites de Internet para pasar a formar parte de otros entornos cotidianos. Es el caso del sistema de televisión del Metro de Madrid, que utiliza Director para la elaboración de composiciones multimedia en las que se embeben los videos que emiten. El problema del uso de estas tecnologías reside en que constituyen un formato cerrado y propietario cuya estandarización se complica por el hecho de que hasta para su reproducción se necesita un software específico de la empresa que lo fabrica. Si obviamos problemas de otro calado y nos centramos en la recuperación de este tipo de recursos, podemos comprobar que, en el mejor de los casos, se puede obtener un sistema de descripción muy somero que al final va a resultar de poca utilidad en una Internet que marcha hacia el enriquecimiento semántico de los recursos que la conforman.

\section{EI Synchronized Multimedia Integration Language (SMIL): una propuesta del World Wide Web Consortium}

El papel del Synchronized Multimedia Integration Language (SMIL) en todo este juego es el de proporcionar un lenguaje, por un lado, lo suficientemente potente para la elaboración de documentos multimedia y, a la vez, absolutamente estandarizado de cara a su integración con las tecnologías existentes y con el desarrollo de una Web Semántica lo más autodefinida posible. Se trata, por tanto, de un esfuerzo por parte del Wide Web Consortium (W3C) de ampliar los

Scire. $10: 2$ (jul.-dic. 2004) 87-97. 
lenguajes de marcado para que den servicio a las nuevas necesidades a las que se enfrentan los usuarios de la World Wide Web (WWW).

No es la intención de este trabajo la de definir, ni siquiera de forma resumida, los elementos que componen SMIL, ya que para esta labor existen varios documentos del W3C especialmente escritos con este objetivo. Tampoco traduciremos aquí las recomendaciones del Consorcio, ya que pretendemos ir más allá de la mera descripción y analizar este lenguaje desde la perspectiva del documentalista. Por esta razón nos centraremos única y exclusivamente en el modulo de metadatos de SMIL 2.0 y en la capacidad del lenguaje para combinarse con otras tecnologías XML a las que el propio SMIL pertenece. No obstante, introduciremos brevemente el lenguaje para contextualizar las reflexiones siguientes.

SMIL es un lenguaje de marcado que pertenece a la familia de XML, y constituye en si una recomendación (Jacobs, 2001) del Consorcio para la creación de documentos multimedia y la sincronización de los distintos elementos que los componen. El lenguaje se compone de nueve módulos dentro de los cuales se especifican las funcionalidades que resuelven las necesidades que surgen en la creación de documentos que poseen un desarrollo temporal:

- Layout: Permite controlar el posicionamiento en pantalla y en los canales de audio de cada uno de los elementos que componen el documento.

- Content Control: Permite seleccionar y controlar un contenido específico.

- Structure: Se encarga de unir todos los módulos. Podríamos decir que se trata de la cola de enganche para los otros módulos.

- Metainformation: Permite especificar una serie de metadatos sobre el contenido.

- Timing and synchronisation: Se trata del corazón del lenguaje, ya que se encarga de la sincronización de medios.

- Linking: Está especialmente diseñado para definir los esquemas de navegación hipermedia tanto dentro del documento como entre documentos.

- Media object: Se trata del modulo encargado de definir el medio de base integrado en una presentación

- Time manipulations: Permite manipular el tiempo, acelerar, saltar, etc.

- Transition effects: Permite definir efectos de realización como fondos, efectos visuales, transiciones, etc.

\section{El modulo de Metadatos de SMIL}

Como hemos mencionado al principio, vamos a centrar nuestra atención en el módulo de metadatos, ya que es el que consideramos de mayor interés de cara 
al documentalista. Esta descripción se basa fundamentalmente en la traducción de la documentación del W3C disponible en su sitio y en el texto de Francisco López Ortiz (2001). Aunque en SMIL, al igual que en los documentos HTML, se puede utilizar la ya antigua etiqueta $<$ meta $>$, el avance en los últimos años en materia de metadatos ha permitido que en la versión actual de SMIL, la 2.0, se haya desarrollado un módulo de metadatos mucho más completo. En SMIL, cada elemento <meta> especifica un único par de propiedad y valor en los atributos de nombre y contenido respectivamente. Estos atributos son cuatro: content, que especifica el valor de la propiedad definida en el elemento meta y es requerido por los elementos meta; id, un identificador de XML que dentifica un elemento dentro de un documento; name, que identifica la propiedad definida en el elemento meta y es también requerido por los elementos meta; $\mathrm{y}$, finalmente, skip-content. Esta última propiedad se introdujo para proporcionar extensibilidad a SMIL en el futuro con las siguientes especificaciones: Si un nuevo elemento es introducido en una futura versión de SMIL y ese elemento permite elementos SMIL 1.0 como elementos de contenido, el atributo skip-content controla si dicho contenido es procesado por un reproductor SMIL 1.0. Si un elemento vacío en la versión SMIL 1.0 se convierte en no vacío en una versión futura de SMIL, controla si este contenido es ignorado por un reproductor SMIL 1.0 o se produce un error de sintaxis. Si el valor del atributo es verdadero (true) y es de aplicación uno de los casos arriba señalados, el contenido del elemento es ignorado. Si el valor es falso (false), el contenido del elemento es procesado. El valor por defecto es verdadero.

La lista de propiedades no está cerrada, y por el momento está formada por las tres siguientes: a) base, que determina el URI base para todas las URI's utilizadas en el documento; b) pics-label o PICS-Label, que especifica una etiqueta válida para el documento según lo definido por PICS (Miller, 1996); y c) title, que contiene el título de la presentación y extiende el elemento meta de SMIL 1.0 con tres atributos, a saber [118N] xml:lang, que especifica el idioma utilizado, http-equiv, que puede ser utilizado en lugar del atributo name para que los servidores HTTP obtengan la información necesaria para construir las cabeceras de los mensajes de respuesta http, y scheme, que da nombre al esquema que se utiliza para interpretar el valor de la propiedad. Al ser el elemento meta un elemento vacío, no contiene información más allá de la que se incluye en los valores de sus atributos.

Como se ha venido demostrando en los últimos años, la capacidad semántica de los metadatos debe ser combinada con elementos sintácticos que definan un entorno de descripción válido. En ese sentido RDF permite un tratamiento más general de los metadatos, ya que se trata de un lenguaje declarativo que proporciona una manera estándar de utilizar XML para representar metadatos en forma de declaraciones acerca de propiedades y relaciones entre elementos de la Red. Dichos elementos, conocidos como recursos, pueden ser casi cualquier

Scire. $10: 2$ (jul.-dic. 2004) 87-97. 
cosa, siempre y cuando tengan una dirección web. Esto significa que es posible asociar metadatos con documentos SMIL, pero también gráficos, archivos de audio, películas, etc.

Resource Description Framework (RDF) es el lenguaje apropiado para la codificación de metadatos (Miller, 2004), ya que los metadatos dentro de un documento SMIL deben ser expresados con los "espacios de nombre" (XMLNS) apropiados (Bray, 1999), y deben ser emplazados dentro del elemento $<$ metadata $>$ hijo del documento raíz de <smil $>$. Por este motivo, RDF parece ser la forma ideal de soportar esquemas de descripción simultánea. A la hora de decidirse por un esquema de metadatos en nuestros documentos deberemos referirnos a las recomendaciones sobre metadatos del W3C RDFsyntax (Manola, 2004) y RDFschema (Brickley, 2004). Además podemos recurrir a la Dublin Core Metadata Initiative (DC) (2005), que es un núcleo de propiedades de metadatos de aplicación general, como título, autor, tema, descripción, etc. Por lo que hace al video, podremos referirnos al Video Metadata Representation, en A Comparison of Schemas for Dublin Core-based Video Metadata Representation (Hunter, 2005), que extiende las propiedades de Dublin Core hasta alcanzar los requerimientos de los metadatos sobre contenidos de video, tales como tipo, relación, formato, cobertura, etc. Por último, el SMIL Metadata Schema (Michel, 2000) contiene un conjunto de propiedades adicionales de metadatos que son comunes a la mayor parte de los usuarios de multimedia. Cualquier creador individual o empresa puede crear sus propios esquemas de metadatos, aunque desde el W3C se promueve la utilización de esquemas estándar siempre que se pueda para aumentar las posibilidades de intercambio e interoperabilidad. En cualquier caso siempre es preferible definir un esquema de metadatos adicional en RDF en combinación con el esquema de metadatos estándar que utilizar un esquema completamente nuevo. A continuación mostramos un ejemplo de como se pueden incluir los metadatos dentro de un documento SMIL. Este ejemplo usa Dublin Core version 1.0 RDF schema DC (Dublin Core Metadata Initiative, 2005) y un hipotético SMIL metadata RDF Schema:

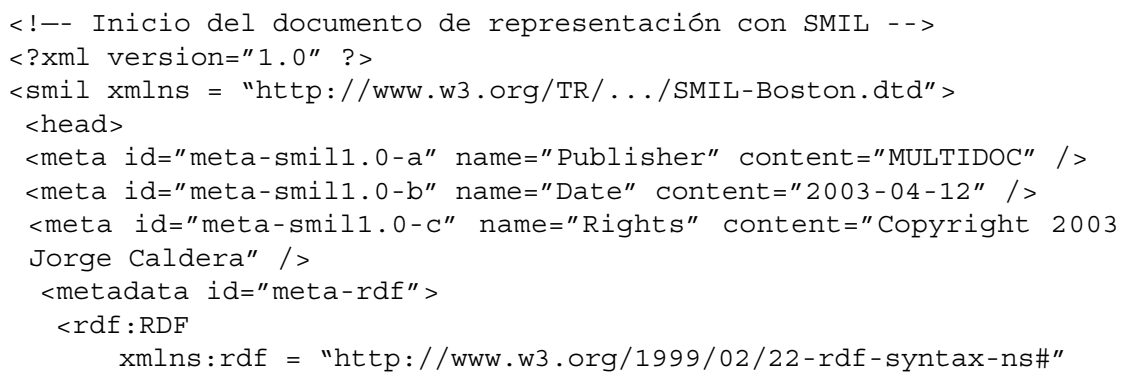

Scire. $10: 2$ (jul.-dic. 2004) 87-97. 
xmlns:rdfs = "http://www.w3.org/TR/1999/PR-rdf-schema19990303\#"

xmlns:dc = "http://purl.org/metadata/dublin_core\#"

xmlns:smilmetadata = "http://www.w3.org/AudīoVideo/.../smilns\#" >

<!-- Metadatos sobre la representación del documento con SMIL -->

El primer paso a la hora de crear un documento que contenga la representación de un archivo audiovisual es describir ese mismo documento, ya que la representación conforma en sí misma un documento que deberemos también procesar y recuperar en un momento determinado. Para ello procedemos de la siguiente forma:

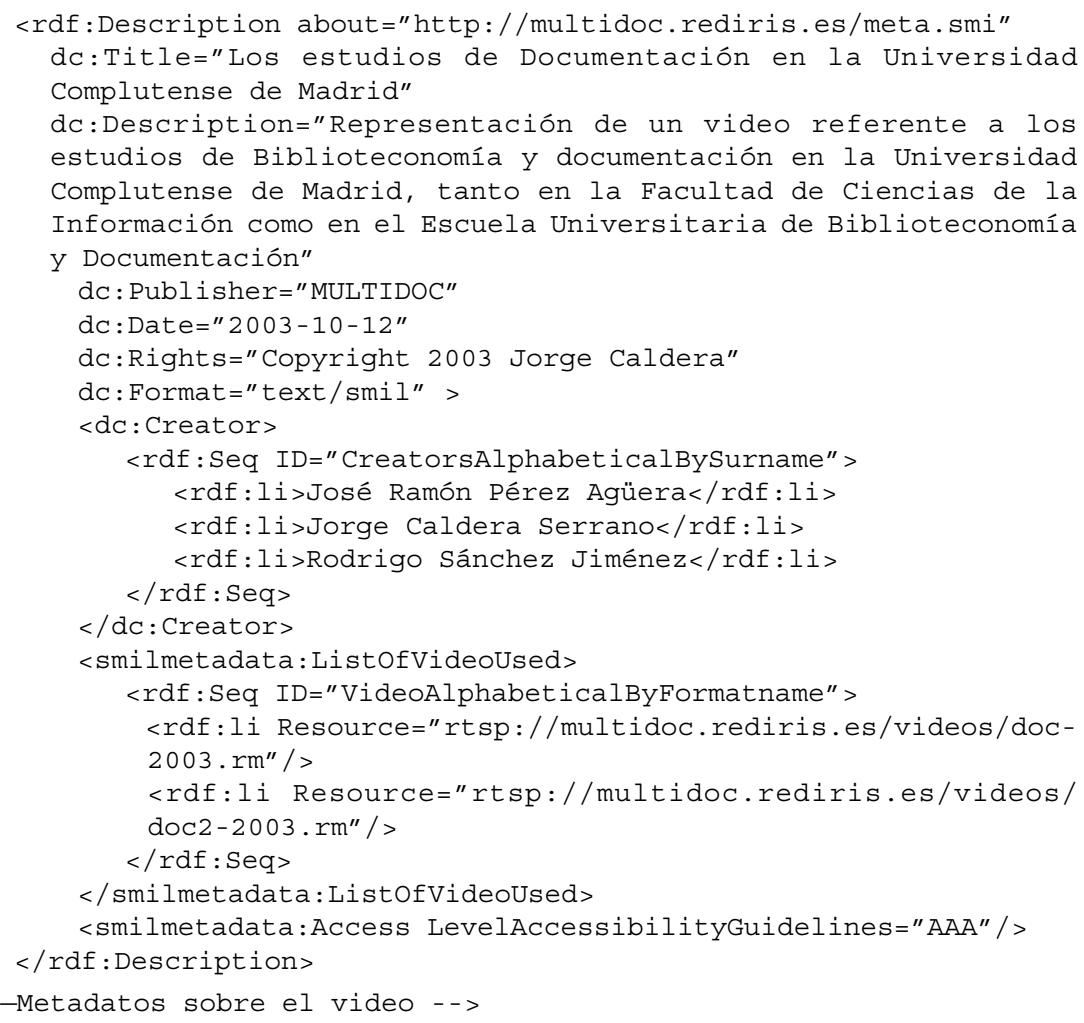

Una vez hemos asignado al documento encargado de representar el archivo audiovisual con el que vamos a trabajar la metainformación necesaria para su posterior procesamiento, podemos empezar a asignar metainformación al archivo en sí, en una primera descripción general que nos ayude a procesar el documento audiovisual en su conjunto.

Scire. $10: 2$ (jul.-dic. 2004) 87-97. 
$<$ rdf:Description about="rtsp://multidoc.rediris.es/videos/doc$2003 . r m "$

dc:Title="La documentación en la Universidad Complutense de Madrid. $1^{\text {a }}$ parte"

dc: Creator="Alfonso López Yepes"

dc: Subject=" Documentación, Formación"

dc: Description="Descripción de los estudios de biblioteconomía y documentación en la UCM"

dc: Publisher="Servicio de Documentación Multimedia (MULTIDOC)"

dc $:$ Format ="video/ $\mathrm{rm}^{\prime \prime}$

dc $:$ Language $="$ es"

dc $:$ Date $=" 2003-04-12 "$

smilmetadata:Duration=" 60 secs"

smilmetadata:VideoCodec="RealMedia" >

<smilmetadata: ContainsSequences >

<rdf:Seq ID="Chronologicalsequences" >

<rdf:li Resource="rtsp://multidoc.rediris.es/videos/doc2003.rm\#scene1" / >

<rdf:li Resource="rtsp://multidoc.rediris.es/videos $/$ doc -

2003 .rm\#scene2" / >

$</$ rdf : Seq $>$

$</$ smilmetadata: ContainsSequences $>$

$</$ rdf : Description $>$

$<$ !-Metadatos sobre una escena del video -->

Tras describir el video en su conjunto podemos establecer tantos niveles de detalle como queramos, para facilitar así la recuperación de los documentos en función del tratamiento de las distintas partes que lo componen. Un ejemplo de esto se proporciona a continuación, donde describimos una escena del video que hemos analizado con anterioridad para así poder referirnos a ella cuando queramos y recuperarla en caso de ser necesario.

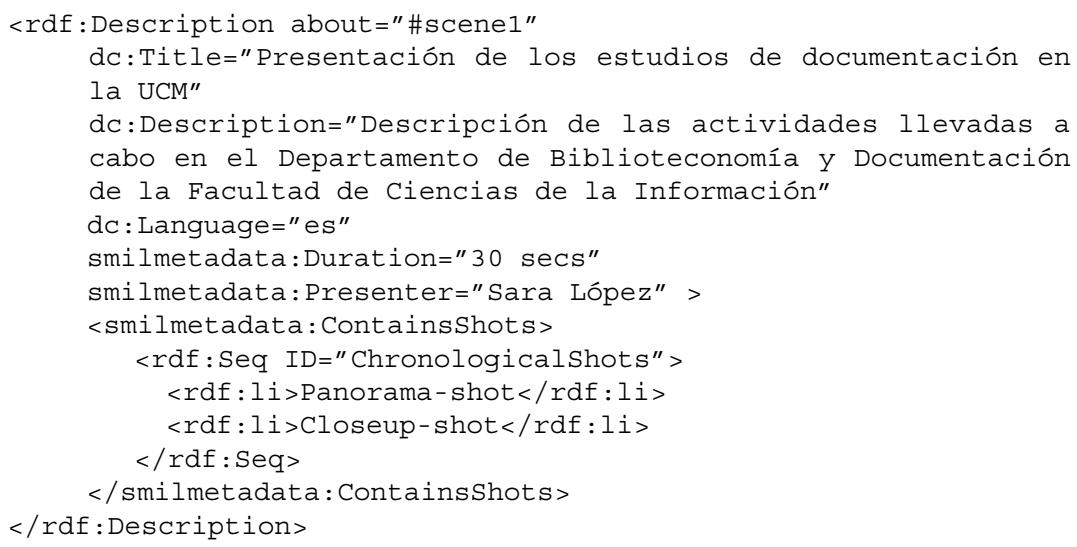

Scire. $10: 2$ (jul.-dic. 2004) 87-97. 


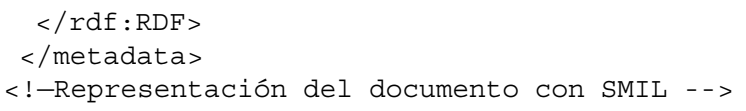

Una vez hemos realizado la descripción del documento tanto en sus aspectos formales como conceptuales gracias a la utilización de metadatos, diseñamos la forma en la que la representación será mostrada en pantalla al usuario.

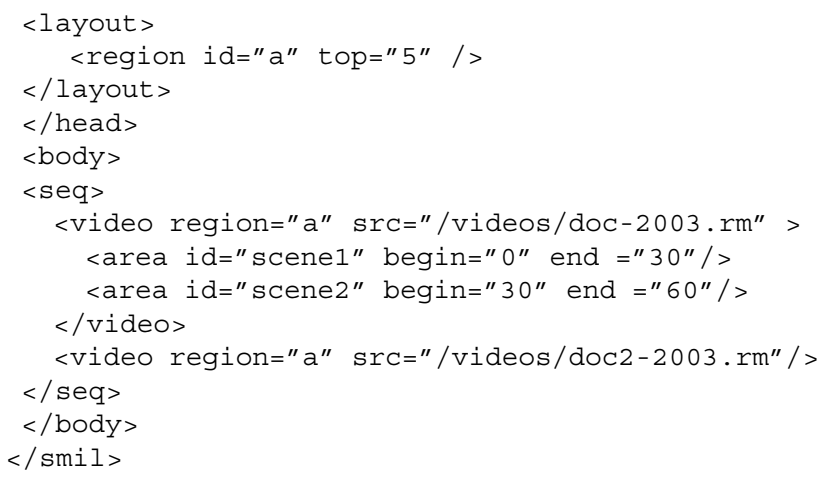

\section{Conclusiones}

SMIL permite la creación de un entorno unificado de descripción de documentos multimedia y de los elementos que los componen independientemente de su formato. Gracias a SMIL podemos realizar un tratamiento natural e integrado de la multicanalidad (Pinto Molina, García Marco y Agustín Lacruz, 2002). Existe la posibilidad de enlazar videos y partes de videos, pudiéndose adaptar esta funcionalidad fácilmente a entornos de descripción de video, como es el caso de los minutados en centros de documentación en televisión. El mismo documento puede dar lugar a infinitud de nuevos documentos sin duplicación de sí mismo ni pérdida de su integridad. La flexibilidad que permite SMIL en la descripción del documento aumenta enormemente su recuperabilidad y la de sus elementos componentes, lo que permite a su vez un mayor aprovechamiento de la interactividad que caracteriza a los documentos multimedia.

El hecho de que cada elemento puede ser descrito por sí mismo en el documento del que forma parte permite su recuperación en función de los documentos en los que aparece sin necesidad de que este dato figure de forma independiente, optimizando el volumen de información que aparece sobre un recurso y permitiendo una recuperación contextual en función del número de discursos construidos con ese documento.

Si entendemos un documento como un recurso que puede ser utilizado más allá de las funciones comunicativas para las que se pensó primitivamente 
(Pinto Molina; García Marco; Lacruz, 2002), SMIL permite esta utilización del documento manteniendo su unicidad y su coherencia en si mismo, sin que esto produzca una interferencia en el nuevo documento que se está componiendo. Así pues, la enumeración de los conceptos que realmente contiene el documento y aquellos que le son asignados de forma concreta en uno de sus usos potenciales pueden aparecer en el mismo documento aumentando la capacidad de precisión con la que podemos describir un recurso, lo cual permite una mayor capacidad de recuperación aumentando el grado de exhaustividad sin que la precisión en la recuperación se vea afectada. Por ejemplo, supongamos que tenemos un video como parte integrante de un documento multimedia, este video como documento con sentido en si mismo contiene uno términos de indización concretos que expresan los conceptos contenidos en este documento de cara a su posterior recuperación. Ahora bien, el documento multimedia que contiene este video como una de sus partes integrantes tiene asignados a su vez unos términos de indización que al igual que en el caso anterior expresan los conceptos contenidos en ese documento. Estos segundos términos de indización pertenecientes al documento multimedia describen el documento multimedia como un conjunto, pero en ningún caso sirven para describir cada una de sus partes. Ahora bien, el hecho de contar con estos términos de indización unidos a los términos de indización que se han asignado a cada uno de los recursos que componen el documento permite la combinación de ambos de cara de dotar al documento multimedia y a los que lo componen de una relevancia específica en función a una consulta efectuada. Esto quiere decir que se puede recuperar el video de nuestro ejemplo no solamente a través de los términos de indización que se le han asignado, sino también de aquellos asignados a los documentos de los que forma parte, aumentando así la precisión de la respuesta a la búsqueda sin que la exhaustividad se vea afectada de forma real. Podríamos decir que los términos de indización asignados al documento multimedia nos sirven para contextualizar el video de cara a una necesidad de recuperación específica. Así pues, podemos recuperar un documento no solamente en función de los conceptos que contiene, sino también en función del contexto en el que se utiliza reflejado por el documento multimedia que lo contiene. Si llevamos este ejemplo a un caso práctico podríamos imaginar la siguiente situación: En el centro de documentación de una cadena de televisión se necesita recuperar videos sobre amaneceres que hayan sido utilizados en anuncios de campañas electorales. En el sistema actual la indización de esta recuperación sólo es factible a partir de la recuperación de los términos de indización contenidos por el video de propaganda electoral, donde no tiene necesariamente por qué aparecer el término amanecer, ya que no se refiere a ninguno de los conceptos expresados o contenidos en el documento, y sería el minutado el encargado de poner en relación la puesta de sol con el documento de propaganda electoral que lo contiene.

Scire. $10: 2$ (jul.-dic. 2004) 87-97. 
Esto supone un minutado implícito, ya que permite a su vez la sincronización de las descripciones de esos medios, eliminando la necesidad de repetir trabajo y presentando a SMIL como una sintaxis válida para la realización de minutados. Podríamos decir que SMIL permite asignar una estructura secuencial tanto al documento multimedia como a su descripción documental. Además, al ser SMIL un lenguaje XML permite aprovecharse de toda la potencia y flexibilidad de éste último tanto en la creación de documentos como en su análisis de cara a una posterior recuperación.

Por otra parte, la gran ventaja que ofrecen los lenguajes de marcado en la recuperación de información reside en la posibilidad que brindan de estructurar el contenido de un documento y dotarle de significado gracias a las etiquetas o marcas que se le asignan. Así pues, en el caso de SMIL las marcas que definen secuencias <seq> y los atributos que definen la sincronización de los medios dentro del documento como <seq begin"'"=> pueden utilizarse de forma sencilla para recuperar información a lo largo del desarrollo temporal de un documento, pudiendo dirigirnos no solo a una parte concreta del mismo, sino también a un tiempo concreto dentro del documento, lo cual facilita una variable importante de recuperación de los ítems que tienen desarrollo cronológico. Además, la posibilidad de generación de documentos SMIL de forma dinámica a través de scripts es otra de las grandes ventajas de la utilización de este lenguaje, ya que permite la creación de documentos multimedia "al vuelo" en función de las necesidades concretas de un usuario en un momento dado. Iniciativas como Cuypers (Manniesing, 2005) en Holanda están trabajando en este sentido con resultados ciertamente espectaculares.

En conclusión, SMIL es un estándar del W3C; una tecnología abierta, gratuita y al alcance de todos, flexible y con grandes posibilidades de desarrollo, que hasta el momento está recibiendo el apoyo de grandes empresas como Real Network, Microsoft, Apple, IBM, que están desarrollando software que soporta esta recomendación y participan de forma activa en el desarrollo del estándar.

\section{Referencias}

Ayars, Jeff; Bulterman, Dick; Cohen, Aaron (2005). Synchronized Multimedia Integration Language (SMIL 2.0). [Second Edition]. URL: <http://www.w3.org/TR/2005/RECSMIL2-20050107 >. Consultado: Enero 2005.

Bray, Tim (1999). Namespaces in XML. URL: <http://www.w3.org/TR/REC-xml-names/>.

Brickley, Dan (2004). RDF Vocabulary Description Language 1.0: RDF Schema. URL: <http://www.w3.org/TR/rdf-schema/ >.

Dublin Core Metadata Initiative (2005). DCMI term declarations represented in RDF schema language. URL <http://dublincore.org/schemas/rdfs/>. Consultado: Enero 2005 .

Scire. $10: 2$ (jul.-dic. 2004) 87-97. 
HELIO (1999). Tutorial de SMIL 2.0. URL: <http://www.helio.org/products/smil/tutorial/>. Consultado: Enero 2005.

Hunter, Jane (2005). A Comparison of Schemas for Dublin Core-based Video Metadata Representation. URL: <http://archive.dstc.edu.au/RDU/staff/jane-hunter/mpeg7/ contribution.html>. Consultado: Enero 2005.

Jacobs, Ian (2001). World Wide Web Consortium Process Document. URL: <http:// www.w3.org/Consortium/Process-20010719/process.html\#RecsW3C>. Consultado: Enero 2005.

López Ortiz, Francisco (2001). SMIL 2.0: Una solución XML para Streaming. URL: <http:/greco.dit.upm.es/ tomas/cursos/isi/trabajos/2001/smil2-0.doc >. Consultado: Enero 2005.

Michel, Thierry (2005). Home Page de Synchronized Multimedia del W3C. URL: <http: //www.w3.org/AudioVideo/ >. Consultado: Enero 2005.

Miller, Jim (1996). PICS Label Distribution Label Syntax and Communication Protocols. URL: <http://www.w3.org/TR/REC-PICS-labels >.

Miller, Jim (2004). Resource Description Framework (RDF). URL: <http://www.w3.org/ $\mathrm{RDF} />$.

Manola, Frank (2004). RDF Primer. URL: <http://www.w3.org/TR/REC-rdf-syntax/>.

Manniesing, Amit (2005), Color selection demo. URL: <http://homepages.cwi.nl/ media/ demo/cuypers/>.

Michel, Tierry (2000). The SMIL Metadata Module. URL<http://www.w3.org/TR/2000/ WD-smil-boston-20000225/metadata.html\#-metadata>.

Newman, Debbie; Patterson, Aaron; Schmitz, Patrick; (2002). Perfil XHTML+SMIL. URL: <http://www.w3.org/TR/XHTMLplusSMIL/ > . Consultado: Enero 2005.

Newman, Debbie (2000). Spice Up Your Web Pages with HTML+TIME. URL: <http: $/ / \mathrm{msdn}$.microsoft.com/library/en-us/dntime/html/htmltime.asp >. Consultado: Enero 2005.

Pinto Molina, María ; García Marco, Francisco Javier ; Agustín Lacruz, María del Carmen (2001). Indización y resumen de documentos digitales y multimedia : técnicas y procedimientos. Gijón: Trea, 2001.

Viken, Asa (2001). Streaming: Past, Present and Future: An Investigation into the Synchronized Multimedia Integration Language 2.0 (SMIL 2.0). Tesis doctoral. Estocolmo: Royal Institute of Technology, 2001.

Wor Wide Web Consortium. Cambridge: MIT; Sophia Antipolis: ERCIM; Tokio: Keio, 1994-2005. URL: <http://www.w3c.org>. 